Please cite as: Rudert, S. C., \& Greifeneder, R. (2018). Bedrohung der Zugehörigkeit: Soziale Ausgrenzung in Organisationen. In O. Geramanis \& S. Hutmachter (Eds.), Identität in der modernen Arbeitswelt (pp. 49-66). Heidelberg: Springer.

\title{
Bedrohung der Zugehörigkeit: Soziale Ausgrenzung in Organisationen
}

Selma Carolin Rudert and Rainer Greifeneder

Fakultät für Psychologie

Abteilung Sozialpsychologie

Universität Basel

Beitrag zur Publikation in

Identität in der modernen Arbeitswelt (Springer) 


\begin{abstract}
Die Zugehörigkeit zu sozialen Gruppen ist ein zentrales menschliches Bedürfnis. Die Bedeutsamkeit der Zugehörigkeit zeigt sich beispielsweise darin, dass bereits die kleinsten Anzeichen davon, ausgeschlossen zu sein oder ignoriert zu werden, dazu führen, dass Menschen sich in ihrer Zugehörigkeit bedroht und verletzt fühlen. Wenn Menschen häufig ausgegrenzt werden, kann dies schwerwiegende Folgen nach sich ziehen, wie Depressionen, Motivationsverlust und ,innere Kündigung“, sowie Aggression und schädigendes Verhalten gegenüber dem Unternehmen und den Mitarbeitenden.

In Organisationen ist Ausgrenzung ein häufiges Phänomen und kann verschiedene Gründe haben: Zum einen kommt es vor, dass Mitarbeitende Andere ausgrenzen, um sie für ein vermutetes Fehlverhalten und Verstösse gegen Normen der Organisation zu bestrafen. So werden beispielsweise „Whistleblower“ oft von anderen Mitarbeitenden ausgeschlossen. Zum anderen kommt es aufgrund von Hierarchien und Arbeitsteilungen in Organisationen häufig zu rollen- und normbedingtem Ausschluss. Dies kann von den Mitarbeitenden in Abhängigkeit von ihrem Verständnis der organisationalen Normen unterschiedlich wahrgenommen und akzeptiert werden. Insbesondere in grossen Organisationen kann es zudem geschehen, dass Mitarbeitende bei Absprachen oder der Informationsweitergabe schlichtweg übersehen werden. Besonders Zeitarbeitende sind von dieser Form des Ausschlusses häufig betroffen.

Die Vielzahl an Auslösern und die drastischen Folgen von wahrgenommener Ausgrenzung unterstreichen die Bedeutsamkeit des Zugehörigkeitsgefühls in Organisationen. Investitionen in das Zugehörigkeitsgefühl können sich somit nachhaltig positiv sowohl auf das Wohlbefinden des einzelnen Organisationsmitglieds als auch auf die Effizienz der Organisation als Ganzes auswirken. Dieser Beitrag diskutiert unter anderem mögliche Wege das Zugehörigkeitsgefühl zu stärken und die Risiken sozialen Ausschlusses durch geeignete organisationale Massnahmen zu reduzieren.
\end{abstract}


Die Zugehörigkeit zu sozialen Gruppen ist ein zentrales menschliches Bedürfnis (Baumeister \& Leary, 1995). Ein Grund hierfür liegt in der evolutionär bedingten, sozialen Natur der menschlichen Spezies: Seit jeher leben Menschen in Gemeinschaften und Gruppen und betreiben Arbeitsteilung. Aus evolutionärer Perspektive hatten Einzelgänger und aus der Gemeinschaft ausgeschlossene Individuen ein hartes Leben und fanden häufig den Tod, da sie den Gefahren der Umwelt alleine ausgesetzt waren. Aber auch in der heutigen Zeit bringt Zugehörigkeit Vorteile: Zu einer Organisation zugehörige Individuen geniessen oftmals Schutz und bestimmte Privilegien, wie Zugriff auf materielle wie immaterielle Ressourcen der Gruppe oder Organisation.

Die hohe Bedeutsamkeit von Zugehörigkeit hat zur Folge, dass Menschen Ausgrenzung als sehr negativ empfinden und im Regelfall versuchen, entsprechende Erfahrungen zu vermeiden (Williams, 2009). Um einen Ausschluss aus der Gruppe abwenden zu können, sind Menschen hochgradig sensibel für Anzeichen, dass sie ausgegrenzt oder ignoriert werden. So fanden beispielsweise Wesselmann, Cardoso, Slater und Williams (2012) in einer Untersuchung auf einem Universitätscampus, dass Studierende bereits verringerte Zugehörigkeitsgefühle berichteten, wenn zuvor eine fremde Person nur an ihnen vorbeiblickte, anstatt ihnen in die Augen zu sehen. Diese hohe Sensibilität führt dazu, dass Akte der Ausgrenzung im Organisationskontext, wie etwa ein Auslassen aus dem E-Mail Verteiler oder ein fehlender Morgengruss, schnell als bedrohlich eingeschätzt werden und mit negativen Gefühlen verbunden sein können. Solche Situationen sind dabei keine Seltenheit: Studien im Arbeitskontext zeigen, dass ein Grossteil der befragten US-amerikanischen Angestellten von zumindest einigen Ausgrenzungserlebnissen in den vergangenen Monaten berichten (Hitlan \& Noel, 2009). 60 - 70\% der Befragten erinnerten sich, ein oder zweimal nicht zu arbeitsbezogenen Aktivitäten eingeladen worden zu sein, keine Rückmeldung von einem Vorgesetzten erhalten zu haben, von anderen Mitarbeitenden wie Luft behandelt oder aus Konversationen ausgeschlossen worden zu sein. An die 7\% berichteten sogar, dass diese 
Formen von Ausgrenzung oft oder sehr häufig vorkämen. Im Folgenden diskutieren wir, welche gravierenden Konsequenzen ein durch Ausgrenzung bedrohtes Zugehörigkeitsgefühl nach sich ziehen kann.

\section{Folgen des bedrohten Zugehörigkeitsgefühls}

Wenn Menschen ausgeschlossen oder ignoriert werden, empfinden sie im ersten Moment ein warnsignalartiges Gefühl von Schmerz (Williams, 2009). Studien zeigen, dass in diesem Moment ähnliche Hirnareale wie bei der Verarbeitung von physischem Schmerz aktiviert werden (Eisenberger, Lieberman, \& Williams, 2003). Zudem berichten Personen unmittelbar nach dem Ausschluss Gefühle von Zugehörigkeits- und Kontrollverlust, verringertem Selbstwert und Bedeutungslosigkeit (Williams, 2009). Studien im organisationalen Kontext zeigen, dass Ausgrenzung am Arbeitsplatz mit Angst, depressiven Gefühlen, emotionalem Stress, verringerter Arbeitszufriedenheit und weniger Verbundenheit und Identifikation mit der Organisation einhergeht (Ferris, Brown, Berry, \& Lian, 2008; Hitlan, Kelly, Schepman, Schneider, \& Zárate, 2006). Neben den psychologischen Konsequenzen für das Individuum gibt es häufig auch negative Konsequenzen für die Organisation: Zum einen hemmt Ausgrenzung eine Person in ihrer Produktivität, da sie Zugriff auf aufgabenbezogene Ressourcen, Beziehungen und Informationen verliert. Dies reduziert die Wahrscheinlichkeit, dass die ausgegrenzte Person eine gute Leistung zeigen, oder im Extremfall sogar, dass sie ihre Arbeit überhaupt erledigen kann (Robinson, O’Reilly, \& Wang, 2013). Zum anderen kann allein die Beobachtung, dass eine Kollegin oder ein Kollege ausgegrenzt wird, negative Empfindungen und ein Gefühl der Bedrohung hervorrufen, ein Phänomen, das als „stellvertretende Ausgrenzung“ bezeichnet wird (Wesselmann, Williams, \& Hales, 2013). Mögliche Gründe hierfür sind, dass die Beobachtenden sich in die ausgegrenzte Person hineinversetzen und mit ihr mitfühlen, oder aber auch, dass sie konkrete Befürchtungen hegen, dass ihnen dasselbe zustossen könnte. 
Welche Reaktionen Menschen auf Ausgrenzung zeigen, hängt stark von den situativen Bedingungen ab (Smart Richman \& Leary, 2009). Wenn Menschen ihre Mitarbeitenden und das Unternehmen sehr schätzen, ein Ausschluss für sie sehr negativ wäre oder sie glauben, die Beziehung zu ihren Mitarbeitenden reparieren zu können, bemühen sie sich wahrscheinlich um die Gruppe und möchten den drohenden Ausschluss abwenden. Anders sieht es aus, wenn sich Alternativen zum aktuellen Job anbieten, die Organisation dem Individuum nicht essentiell wichtig ist, oder wenn die Beziehung zu den Ausgrenzenden irreparabel geschädigt ist. In diesem Fall steigt die Wahrscheinlichkeit, dass eine Person sich zurückzieht und entweder innerlich oder tatsächlich kündigt. Entsprechend konnten in Studien Zusammenhänge zwischen Ausgrenzung am Arbeitsplatz und einer verminderten Leistung der Arbeitnehmenden gezeigt werden (Wu, Wei, \& Hui, 2011). Falls Ausgrenzung zudem auch noch als hochgradig unfair angesehen wird, ist es möglich, dass Menschen aggressiv reagieren und versuchen, ihren Mitarbeitenden oder der Organisation zu schaden beispielsweise in Form einer Klage oder in kontraproduktivem Arbeitsverhalten, wie etwa dem Zurückhalten relevanter Information, absichtliches Verzögern von Arbeitsprozessen, Prokrastination, falschen Krankmeldungen oder Diebstahl (Gkorezis \& Bellou, 2016; Hitlan \& Noel, 2009). Werden Menschen häufig und über einen langen Zeitraum ausgegrenzt und haben keine Möglichkeit, etwas an der Situation zu ändern, beispielsweise weil eine Kündigung aus finanziellen oder arbeitsmarkttechnischen Gründen nicht möglich ist, hat dies oft sehr negative Konsequenzen: Die betroffenen Personen resignieren, werden unmotiviert, verlieren die Freude an ihrer Arbeit und können langfristig sogar Depressionen entwickeln (Riva, Montali, Wirth, Curioni, \& Williams, 2016).

\section{Gründe für soziale Ausgrenzung in Organisationen}

Sowohl für das Wohlbefinden der ausgegrenzten Person, als auch für die Produktivität der Organisation, können durch sozialen Ausschluss negative Konsequenzen entstehen. Warum ist Ausgrenzung innerhalb von Organisationen dennoch ein so häufig auftretendes 
Phänomen? Im Folgenden diskutieren wir die folgenden drei Hauptgründe: Erstens wird Ausschluss oft als bewusste Bestrafung aufgrund eines wahrgenommenen Fehlverhaltens der ausgeschlossenen Person verwendet. Zweitens kommt es in den meisten Organisationen durch bestehende Hierarchien und Formen der Arbeitsteilung zu rollen- oder normenbedingtem Ausschluss. Zuletzt tritt insbesondere in grösseren Organisationen häufig unbewusster Ausschluss auf, bei dem Mitarbeitende bei Absprachen oder der Informationsweitergabe unabsichtlich nicht beachtet oder berücksichtigt werden.

Bestrafender Ausschluss. Die meisten Menschen empfinden Ausschluss, der scheinbar ohne Gründe geschieht, als eine Verletzung geltender sozialer Normen und unfaires Verhalten von Seiten der Ausgrenzenden (Rudert \& Greifeneder, 2016). Anders verhält es sich jedoch, wenn Menschen zu dem Schluss kommen, dass der Ausschluss gerechtfertigt ist, beispielsweise weil die Person sich unfreundlich und unverträglich verhalten hat (Hales, Kassner, Williams, \& Graziano, 2016), oder weil sie durch schlechte Leistung eine Belastung darstellt (Wesselmann, Wirth, Pryor, Reeder, \& Williams, 2013). Auch Längsschnittstudien im organisationalen Kontext zeigen, dass insbesondere Personen, die sich respektlos, unhöflich und egozentrisch verhielten, stärker von Ausgrenzung durch ihre Kolleginnen und Kollegen betroffen waren (Scott, Restubog, \& Zagenczyk, 2013). Bestrafender Ausschluss stellt hierbei einen Korrekturmechanismus dar, welcher das ausgegrenzte Individuum dazu bringen soll, sich verträglicher und mehr im Sinne der organisationalen Normen zu verhalten (Williams, 2002). Zweck des Ausschlusses ist somit der Schutz der Gruppe oder Organisation und die Aufrechterhaltung organisationsinterner Normen: Ändert die Person ihr problematisches Verhalten, so endet (idealerweise) auch die Ausgrenzung.

Robinson und Kollegen (2013) vermuten, dass bestrafender Ausschluss häufig dann auftritt, wenn erstens die erforderliche Zusammenarbeit in der Organisation eher niedrig ist, so dass die Mitglieder nicht zwangsläufig miteinander agieren müssen, um ihre Aufgaben zu erledigen. Zudem ist es wahrscheinlich, dass bestrafende Ausgrenzung eher dann auftritt, 
wenn es wenig andere formalisierte Möglichkeiten gibt, um Konflikte zu lösen. Dies ist beispielsweise der Fall bei einer Organisation mit sehr flachen Hierarchien und wenig Machtstruktur, bei der Konflikte nicht durch Kontrolle von oben gelöst werden können, oder wenn die Organisationskultur offene Konflikte stark ablehnt und sanktioniert. In beiden Fällen liegt es nahe, dass Mitglieder auf eher informelle oder subtilere Methoden wie Ausgrenzung zurückgreifen, um ihre Missbilligung des gezeigten Verhaltens auszudrücken. Die implizite Annahme bei bestrafendem Ausschluss ist, dass der mögliche Gewinn für die Organisation höher als die möglichen Kosten ist. Obwohl bestrafende Ausgrenzung aus dieser Perspektive effektiv sein kann, birgt sie gleichzeitig ein hohes Risikopotential. Beispielsweise hat die ausgegrenzte Person nicht in jedem Fall Kontrolle über den Grund des Ausschlusses: möglicherweise ist eine schlechte Leistung gar nicht darauf zurückzuführen, dass ein/e neue/r Angestellte/r faul oder lernunwillig ist - vielleicht hatte sie/er nur noch nicht genug Zeit, sich in die neue Aufgabe einzufinden oder wurde ungenügend eingewiesen. Ein Ausschluss führt in dieser Situation vermutlich zum Gegenteil des gewünschten Effekts, nämlich dass die Person noch weniger versteht, was zu tun ist, und die Motivation verliert, sich die gewünschten Fähigkeiten anzueignen (Lustenberger \& Jagacinski, 2010). Dazu kommt der bereits erwähnte Verlust von wichtigen Ressourcen, Beziehungen und Informationen, was die Arbeitsleistung ebenfalls verringert (Robinson, et al., 2013). Anstatt effektiv zu sein, kann bestrafender Ausschluss somit einen regelrechten Teufelskreis anstossen, in dem die ausgegrenzte Person aufgrund des Ausschlusses noch weniger in der Lage ist, das gewünschte Verhalten zu zeigen, und in Folge noch stärker ausgegrenzt wird.

Ein besonderer Kreis von Personen, die bestrafenden Ausschluss erfahren, sind so genannte „Whistleblower“, also Personen die Missstände und illegale Praktiken innerhalb ihrer eigenen Organisation aufdecken und damit zumindest gegen implizite organisationale Normen verstossen. Whistleblower befinden sich oft in einer schwierigen Lage: Sie sind zum einen dem Zorn des Topmanagements ausgesetzt, das die Whistleblower absichtlich 
ausgrenzen kann, um sie für ihr illoyales Verhalten zu bestrafen. Beispielsweise kann das Topmanagement Whistleblower bei Beförderungen oder Fortbildungen übergehen, sie nicht zu Meetings einladen oder ihnen wichtige Informationen vorenthalten (Miceli \& Near, 1989; Parmerlee, Near, \& Jensen, 1982). Im Gegensatz zu einer direkten Kündigung, die der Whistleblower vor Gericht anfechten könnte, haben solch subtilere Ausgrenzungsmassnahmen für die Ausgrenzenden den Vorteil, dass sie schlechter nachweisbar sind und deshalb weniger wahrscheinlich negative Konsequenzen nach sich ziehen. Auch von ihren Kolleginnen und Kollegen erhalten Whistleblower oft wenig Unterstützung (Mesmer-Magnus \& Viswesvaran, 2005): Mitarbeitende schätzen Whistleblowing häufig wenig, sei es weil sie eine Entlassungswelle oder eine Verschlechterung ihrer Arbeitsbedingungen aufgrund von erhöhter Spannung in der Organisation befürchten, weil sie sich vorwerfen, nicht selbst etwas gesagt zu haben, oder auch weil sie sich schlichtweg von dem Whistleblower betrogen fühlen. Folglich ist es keine Seltenheit, dass Whistleblower sich einer Mauer des Schweigens und der Ausgrenzung innerhalb ihrer Organisation ausgesetzt sehen (Mesmer-Magnus \& Viswesvaran, 2005; Williams, 2002). Neben den bereits diskutierten negativen Folgen für die Arbeitsleistung sowie die psychische Gesundheit der Whistleblower kann solch eine bestrafende Ausgrenzung in besonderem Masse negativ für die Kultur einer Organisation oder im Extremfall sogar die Gesellschaft als Ganzes sein: Wenn Angestellte antizipieren, dass sie für das Aufdecken von Missständen mit Ausgrenzung bestraft werden, wird Schweigen und Wegschauen möglicherweise zum Standard: Anstatt aufzustehen, schweigen Mitarbeitende dann möglicherweise auch im Angesicht von Diskriminierung, beim Nichteinhalten von Umweltrichtlinien bei der Produktion (Williams, 2002) oder bei unsicheren Praktiken im Gesundheitswesen (Gkorezis, Panagiotou, \& Theodorou, 2016). Als Folge davon können illegitime und gefährliche Organisationspraktiken fortgeführt werden. 
Aus diesen und anderen Gründen kann es für Whistleblower sehr wichtig sein, unerkannt zu bleiben. Wenn dies gelingt, braucht es trotzdem einen Schuldigen. Gleiches gilt für viele Situationen, in denen innerhalb der Organisationen Fehler auftreten, ohne dass die verantwortliche Person bekannt ist, oder sogar ohne dass eine bestimmte Person schuld wäre oder den Fehler hätte vermeiden können. In diesen unklaren Situationen gibt es einen weiteren Kreis an Personen, die bestrafendem Ausschluss wahrscheinlich ausgesetzt sind: Personen, die aufgrund ihrer Gesichtszüge kalt und inkompetent aussehen. Dies zeigt eine Studienreihe von Rudert, Reutner, Greifeneder und Walker (2017), in der über vierhundert Studienteilnehmende spontan darüber urteilten, wie akzeptabel es wäre, eine Person mit einem bestimmten Gesicht aus der Gruppe auszuschliessen. Zuvor waren die Gesichter gezielt so verändert worden, dass sie unterschiedlich kühl oder liebenswürdig, kompetent oder inkompetent wirkten. Die Teilnehmenden fanden es am akzeptabelsten, kalt und inkompetent wirkende Personen auszuschliessen, wahrscheinlich weil diese Personen intuitiv als Störenfriede innerhalb einer Gruppe gesehen werden. Nur aufgrund der Wahrnehmung von Gesichtszügen, nicht aufgrund tatsächlichen Verhaltens, wird Ausschluss hier als akzeptabel wahrgenommen. Dies ist deswegen besonders brisant, weil man typischerweise nicht aus dem Gesicht einer Person auf die tatsächlichen Eigenschaften der Person schliessen kann; sprich, wer kalt und inkompetent wirkt, ist nicht notwendigerweise auch kalt und inkompetent. Organisationsmitglieder, deren Gesicht kalt und inkompetent wirken, haben also eine besonders hohe Chance, zum Sündenbock zu werden, selbst wenn sie für den Grund des Ausschlusses gar nicht verantwortlich sind.

Rollen- und Normenbedingter Ausschluss. Nicht jeder Ausschluss Anderer hat direkt eine bösartige oder zurechtweisende Intention. In grösseren Organisationen mit viel Arbeitsteilung kann es beispielsweise zu Ausschluss aufgrund bestimmter Rollenverteilungen oder Normen innerhalb der Organisation kommen (Rudert \& Greifeneder, 2016). So ist beispielsweise ein Meeting nur auf Mitglieder einer speziellen Arbeitsgruppe beschränkt oder 
bestimmte Informationen sind nur für Mitarbeitende auf einer bestimmten Hierarchieebene zugänglich. Dabei trifft rollen- und normenbedingter Ausschluss auch Führungspersonen beispielsweise sind leitende Angestellte häufig explizit von ihrer Teilnahme an Betriebsversammlungen ausgeschlossen. Im Unterschied zu bestrafendem Ausschluss wird rollen- oder normbedingter Ausschluss von den Beteiligten typischerweise nicht als problematisch oder bedrohlich wahrgenommen. So zeigten Rudert und Greifeneder (2016) beispielsweise, dass der Ausschluss aus einem virtuellen Ballwurfspiel nicht schmerzt oder als bedrohlich wahrgenommen wird, wenn die Studienteilnehmenden die Rolle eines Trainers hatten, der das Ballspiel explizit nur beobachten sollte. Dagegen schmerzt der Ausschluss aus diesem Ballspiel sehr, wenn man die Rolle eines normalen Mitspielenden hat oder gerne hätte. Gleichermassen schmerzt sozialer Ausschluss weniger, wenn Individuen sich durch ihre Rolle in einer Situation „oben“ befinden (Schoel, Eck, \& Greifeneder, 2014), zum Beispiel weil sie eine machtvolle Position oder Führungsrolle in der Organisation haben.

Rollen- und normenkonformer Ausschluss kann allerdings dann zum Problem werden, wenn das ausgeschlossene Individuum die entsprechende Norm nicht kennt oder sie auch schlichtweg nicht teilt. Um dies experimentell zu demonstrieren, baten Rudert und Greifeneder (2016) Mitglieder verschiedener deutscher politischer Parteien an einer politischen Onlinedebatte teilzunehmen. Die Hälfte der Teilnehmenden bekam im Vorfeld mitgeteilt, dass sie doch nicht an der Diskussion teilnehmen könnten, da eine ausgeglichene Quote zwischen männlichen und weiblichen Diskussionsteilnehmenden angestrebt werde und es angeblich bereits zu viele Diskutierende mit dem Geschlecht des oder der jeweiligen Teilnehmenden gäbe. Studienteilnehmende, die einer Partei des linken politischen Spektrums angehörten, fühlten sich durch den Ausschluss nicht bedroht, denn diese Spielregel entspricht den Regeln und Normen in ihrer Partei. Parteimitglieder aus dem tendenziell konservativen Parteienspektrum hingegen, die eher wenig von Quotenregelungen halten, zeigten typische negative Reaktionen auf den Ausschluss. Die Bedeutung von Normen zeigte sich auch in 
einer Befragung nach der Schweizer Volksabstimmung „Gegen Masseneinwanderung“ im Jahr 2014: Je stärker befragte Immigrant/innen eine Regulation von Zuwanderung im Allgemeinen ablehnten, umso stärker fühlten sie sich in Folge der Abstimmung persönlich bedroht (Rudert, Janke, \& Greifeneder, 2017).

Die Gefahr bei rollen- und normkonformen Ausschluss liegt somit darin zu übersehen, dass eine Ausschlusssituation, die von den Einen als „,normal“ und unbedenklich angesehen wird, von andersdenkenden Betroffenen als schmerzhaft und unfair empfunden werden kann. Dies kann insbesondere dann der Fall sein, wenn die Organisationskultur recht schwach oder gerade im Wechsel ist, beispielsweise wenn Neueinstellungen an der Tagesordnung sind oder während einer Fusion (Robinson, et al., 2013).

Drücken die Betroffenen ihr Empfinden aus und zweifeln damit die vorherrschende Gruppennorm an, werden sie häufig von anderen Organisationsmitgliedern abgewertet oder sogar bestraft (Pinto, Marques, Levine, \& Abrams, 2010). Es ist denkbar, dass sich die Betroffenen hierdurch noch stärker ausgeschlossen fühlen, da sie zusätzlich zu dem bereits erlebten Ausschluss nun auch noch zusätzliches Unverständnis und Abwertung erfahren. Als Konsequenz bliebe dann nur die Wahl, die als ungerecht empfundene Ausschlussnorm letztlich zu akzeptieren oder aber die Organisation zu verlassen.

Insbesondere neue Organisationsmitglieder, welche mit den spezifischen Ein- und Ausschlussnormen einer Organisation noch unvertraut sind, können normkonforme Ausschlusssituationen möglicherweise schnell als verletzend erleben. Da Menschen hochgradig sensibel auf Anzeichen von Ausgrenzung reagieren (Rudert, Hales, Greifeneder, \& Williams, 2017; Williams, 2009), neigen sie im Zweifelsfall eher dazu, eine Ausschlusserfahrung als intentionalen Akt anzusehen und auf sich persönlich zu beziehen anstatt als belanglos abzutun (Rudert \& Greifeneder, 2016). Im Gegensatz zu langjährigen Mitgliedern einer Organisation, werden Kollegen und Kolleginnen dem neuen Mitglied 
jedoch mit grösserer Wahrscheinlichkeit beistehen und ihm oder ihr die entsprechenden Normen kommunizieren (Pinto, et al., 2010).

Unbewusster Ausschluss. Verwandt mit rollen- und normkonformen Ausschluss ist das Phänomen des unbewussten Ausschlusses (Williams, 2002). Auch hier haben die Ausgrenzenden keine feindseligen oder bestrafenden Intentionen gegenüber der ausgeschlossenen Person. Der Ausschluss ist vielmehr darin begründet, dass die Ausgrenzenden nicht wissen oder nicht wahrnehmen, dass die entsprechende Person existiert. Unbewusster Ausschluss kann sowohl durch Faktoren begünstigt werden, welche in der Struktur der Organisation verankert sind, aber auch durch das Individuum betreffende Faktoren. Insbesondere stressreiche Arbeitsumgebungen, die durch hohe Arbeitsbelastung, enge Fristen oder viele persönlichen Konflikte geprägt sind, setzen die Mitarbeitenden unter hohen Stress und psychologischen Druck (Spector \& Jex, 1998). Dies kann dazu führen, dass versehentlich Mitarbeiter vergessen werden, beispielsweise weil keine Zeit war, um doppelt zu überprüfen, ob alle Personen zum gemeinschaftlichen Apéro eingeladen wurden. Auch hohe geographische Entfernung zwischen den Mitgliedern einer Organisation, die im digitalen Zeitalter keine Seltenheit mehr ist, kann unbewusste Ausgrenzung begünstigen und zu Gefühlen von Isolation und verminderter Zugehörigkeit bei den Betroffenen führen (Golden, Veiga, \& Dino, 2008). So wird etwa vergessen, den Kollegen im anderen Gebäude zu fragen, ob er mit zum Mittagessen möchte, oder die im Homeoffice arbeitende Kollegin wird bei der Absprache für das nächste Meeting nicht berücksichtigt, da dieses spontan auf dem Gang vereinbart wurde. Ähnliches gilt für grosse Organisationen mit vielen Mitgliedern, in denen Einzelpersonen oder zum Teil auch ganze Gruppen unabsichtlich bei einer Rundmail vergessen werden, oder zum Beispiel im Rahmen von Bewerbungsverfahren nicht auf alle Bewerbungen persönlich eingegangen werden kann.

Ein weiterer organisationaler Faktor ist eine hohe Diversität innerhalb der Organisation: Obwohl häufig ein wünschenswertes und angestrebtes Ziel, kann Diversität 
unbewusste Ausgrenzung begünstigen. Dies ist beispielsweise dann der Fall, wenn sehr viele unterschiedliche Sprachen innerhalb der Organisation gesprochen werden und eine Person sich dadurch ausgegrenzt fühlt, dass andere sich in einer Sprache unterhalten, die sie nicht versteht (Hitlan, et al., 2006). Sprachbedingter Ausschluss ist mit hoher Unsicherheit für die Betroffenen verbunden, da sie nur schwer herausfinden können, weswegen sie ausgegrenzt werden. Ausserdem fragen sie sich möglicherweise, ob die Anderen gerade über sie reden. Möglicherweise aus diesem Grund führt sprachbedingter Ausschluss zu einem stärkeren Gefühl der Bedrohung und geringerer Verbundenheit mit der eigenen Arbeitsgruppe als nicht sprachbedingter Ausschluss (Hitlan, et al., 2006). Interessanterweise sollte auch hier gelten: je mehr Multilingualität in einer Organisation, wodurch das Nichtverständnis einzelner Kommunikationen zur Selbstverständlichkeit und Norm wird, desto weniger verletzend sollte sprachbedingter Ausschluss wirken.

Ein weiteres, verwandtes Problem stellt im elektronischen Zeitalter die Verwendung von organisationsinterner Sprache oder Abkürzungen nach, welche gerade für Neulinge oft schwer zu verstehen sind. Neuere Forschung zeigt auch hier, dass der Erhalt von unverständlicher Information oder auch der Verwendung von unklaren Abkürzungen bei Menschen das Gefühl hervorruft, nicht im Bilde und damit ausgegrenzt zu sein (Hales, Williams, \& Rector, 2017; Jones, Carter-Sowell, Kelly, \& Williams, 2009).

Auch bei unbewusstem Ausschluss gibt es besonders gefährdete Personengruppen. Neue Mitarbeitende sind beispielsweise überdurchschnittlich häufig von unbewusstem Ausschluss betroffen, da andere Organisationsmitglieder entweder noch nicht von ihnen wissen oder vergessen sie einzubeziehen. Möglicherweise sind die neuen Mitarbeitende auch schlicht noch nicht ausreichend in die Organisationsstrukturen eingebunden, um am Informationsaustausch voll zu partizipieren. Unbewusster Ausschluss kann sicherlich mit ein Grund dafür sein, warum Neubeginne in einer Organisation von vielen Menschen als hochgradig aversiv erlebt werden (Saks, 1994). 
Eine zweite gefährdete Gruppe sind Minderheiten jeglicher Art, beispielsweise Personen, die sich aufgrund ihres Alters oder ihres ethnischen Hintergrunds als unähnlich zu ihren Kolleginnen und Kollegen empfinden. Im Vergleich zu Personen, die sich einer Mehrheit zugehörig fühlen, erleben Zugehörige einer Minderheit sozialen Ausschluss als aversiver und schmerzhafter (Eck, Schoel, \& Greifeneder, 2016; Eck, Schoel, \& Greifeneder, in press).

Eine dritte gefährdete Gruppe stellen Zeitarbeitende dar, welche nur für eine befristete Dauer in einem Unternehmen angestellt sind. Befragungen und Fallstudien zeigen, dass Zeitarbeitende das Verhalten ihrer dauerhaft angestellten Kollegen oft als unpersönlich und respektlos wahrnehmen und diese sich häufig nicht einmal die Mühe machen, den Namen der Zeitarbeitenden zu lernen (Feldman, Doerpinghaus, \& Turnley, 1994; von Hippel, Mangum, Greenberger, Heneman, \& Jeffrey, 1997). In einer Befragung in Australien in mehreren Organisationen aus den Bereichen Wirtschaft und öffentlicher Dienst (Williams, 2002) berichteten Zeitarbeitende von stärker empfundener Ausgrenzung. Ausgrenzung wiederum war mit einem geringeren Zugehörigkeitsgefühl, weniger Arbeitszufriedenheit und engagement und mehr subjektivem Stress assoziiert. Beispielsweise beklagten sich die Zeitarbeitenden, dass sie häufig nicht zum gemeinsamen Essen eingeladen und nicht von Kollegen in Gespräche einbezogen wurden. Festangestellte Kollegen hingegen berichteten, dass sie es als Verschwendung ihrer Zeit und Energie empfanden, sich länger mit den Zeitarbeitenden zu befassen und sie mit einzubeziehen.

Es ist möglich, dass unbewusster Ausschluss eine der schlimmsten Formen von sozialem Ausschluss darstellt, auch wenn oder gerade weil ihm keine Absicht zugrunde liegt. Neben dem Bedürfnis nach Zugehörigkeit haben Menschen ein inhärentes Bedürfnis danach, zu wissen, dass ihre Existenz bedeutsam ist und Andere sie beachten. Unbewusster Ausschluss greift gleich beide dieser zentralen Bedürfnisse an, insofern dass die Betroffenen nicht nur das Gefühl haben, dass Andere keinen Wert auf ihre Anwesenheit legen, sondern sie 
sogar so unwichtig sind, dass sie der Aufmerksamkeit nicht wert sind. In Befragungen gaben Angestellte an, dass diese es schlimmer fanden, ignoriert und unbeachtet zu sein, als aktiv drangsaliert oder gehänselt zu werden (O'Reilly, Robinson, Berdahl, \& Banki, 2015). Dies zeigt sich auch in einer experimentellen Studie (Rudert, Hales, et al., 2017), in der Studienteilnehmende sich für verschiedene Wohnungen bewarben und von den Mitbewohnenden abgelehnt wurden. Studienteilnehmende erholten sich schneller von der Ablehnung, wenn sie zusätzlich zu einer Standard-Information über die Ablehnung eine kurze persönliche Nachricht von einem der Mitbewohner erhielten. Dies war sogar dann der Fall, wenn diese persönliche Nachricht in einem sehr feindseligen Tonfall geschrieben war und es offensichtlich war, dass der Mitbewohner die sich bewerbende Person nicht mochte.

\section{Präventive Massnahmen zur Vermeidung des Gefühls von Ausgrenzung in}

\section{Organisationen}

In den vorangegangen Abschnitten wurde eine Vielzahl an Faktoren diskutiert, die zu einem Gefühl von sozialem Ausschluss innerhalb einer Organisation beitragen können. Ebenso wurde eine Reihe von psychischen und ökonomischen Folgen aufgeführt, die sich aus dem Gefühl von Ausgrenzung ergeben können. Im Folgenden möchten wir verschiedene Möglichkeiten diskutieren, wie Organisationen dazu beitragen können, das Zugehörigkeitsgefühl ihrer Mitarbeitenden zu stärken und die Risiken sozialen Ausschlusses durch geeignete organisationale Massnahmen zu senken. Dabei können sich die diskutierten Massnahmen nachhaltig positiv sowohl auf das Wohlbefinden des einzelnen Organisationsmitglieds als auch auf die Effizienz der Organisation als Ganzes auswirken. Die hier vorgestellten Massnahmen leiten sich logisch aus bestehender Forschung zu sozialem Ausschluss ab, die jedoch häufig im Labor durchgeführt wurde. Ergebnisse von Feldforschung liegen nur selten vor, so dass die hier vorgestellten Massnahmen prinär spekulativer Natur sind und auf eine empirische Fundierung im Feld warten. Dennoch erscheinen sie uns als hilfreiche Denkansätze. 
Sensibilisierung der Organisationsmitglieder. Eine mögliche Massnahme ist es, die Mitglieder der Organisation über das Auftreten und die gravierenden Folgen von sozialer Ausgrenzung zu sensibilisieren, zum Beispiel im Rahmen von Workshops oder Trainings (Williams \& Nida, 2014). Dies erscheint insbesondere dann angezeigt, wenn in einer Organisation oft von bestrafender Ausgrenzung Gebrauch gemacht wird. Das Ziel ist dann eine Veränderung der vorherrschenden Organisationskultur hin zu einer inklusiven und offeneren Organisation, in der Konflikte angesprochen und geklärt werden können (Gkorezis \& Bellou, 2016). Insbesondere Personen mit Führungsverantwortung sollten dahingehend geschult werden, Konflikte mit ihnen unterstellten Mitarbeitern konstruktiv zu lösen, sowie Ausgrenzung von einzelnen Mitgliedern in ihren Teams zu erkennen und gegebenenfalls offen zu thematisieren (Gkorezis \& Bellou, 2016). Aber auch Organisationen, die ein hohes Potential für unbewussten Ausschluss aufweisen, wie beispielsweise grosse Organisationen mit starker Fluktuation und Diversität, oder Tätigkeitsbereiche mit vielen Stressoren, wie beispielsweise hoher Arbeitsbelastung, engen Zeitrahmen oder internen Querelen, können von entsprechenden Sensibilisierungsmassnahmen profitieren.

Räume für Kritik schaffen. Bestrafende Ausgrenzung wird oft eingesetzt um Normenverstösse zu sanktionieren - in vielen Fällen können diese Normverstösse allerdings wertvoll sein. Dies ist zum Beispiel der Fall, wenn es um Kritik am Unternehmen und dessen Abläufen geht (wie im Beispiel der bereits diskutierten Whistleblower): In vielen Organisationen bleibt Potential für Verbesserungen in den Abläufen oder der Struktur ungenutzt, da die Mitglieder sich entweder gar nicht erst trauen etwas anzumerken, oder aber als Unruhestiftende oder Gefährdende althergebrachter Routinen abgestraft werden (Gkorezis, et al., 2016; Miceli \& Near, 1989). Sozialpsychologische Studien zeigen, dass normativer Gruppendruck sogar so weit gehen kann, dass die Betroffenen eine Aussage unterstützen, von der sie klar wissen, dass sie falsch ist (Asch, 1956). Abhilfe schaffen kann hier eine Organisationskultur, die Vorschläge und Partizipation ihrer Mitarbeitenden nicht nur zulässt, 
sondern auch fördert (Cotton, Vollrath, Froggatt, Lengnick-Hall, \& Jennings, 1988), zum Beispiel durch Mitarbeiterbefragungen, Vorschlagssysteme, oder kontinuierliche Verbesserungsprozesse, in denen Mitarbeitende die konkrete Aufgabe haben, über die Verbesserung von Abläufen in der Organisation zu diskutieren. Aber auch für Kritik im Sinne von Beschwerden sollten Räume geschafft werden, wie beispielsweise bei sexueller Belästigung (Miceli, Near, \& Dworkin, 2009). Hier ist es beispielsweise sinnvoll, eine sichere Anlaufstellen innerhalb der Organisation zu schaffen, an welche das Mitglied sich unter zugesicherter Anonymität wenden kann, welche die entsprechenden Vorfälle prüft und gegebenenfalls Schritte einleitet.

Klärung organisationaler Normen. Jede Organisation hat eine Kultur und zugehörige Normen, doch häufig sind diese Normen nicht explizit formuliert. Damit es nicht aufgrund von Unkenntnis oder Unverständnis der Normen zu Ausgrenzungserfahrungen kommt (Rudert \& Greifeneder, 2016), sollten organisationsinterne Normen transparent und offen dargelegt sowie Neulingen erklärt werden. Neben HowTos und Kickoff Veranstaltungen bieten sich hierfür beispielsweise auch Mentoring Programme an, welche zusätzlich noch einen positiven Effekt auf das Zugehörigkeitsgefühl haben können (Korte, 2009). Der Wert selbst kleiner Massnahmen sollte hierbei nicht unterschätzt werden: In den Studien von Rudert und Greifeneder (2016) zeigte sich, dass selbst geringfügige Unterschiede in den Instruktionen zu bedeutsamen Unterschieden im Wohlbefinden der Ausgeschlossenen führten. Wurde beispielsweise der Teilnehmende in der Trainerrolle darauf hingewiesen, dass er den Trainierenden lediglich zuschauen sollte, fühlte er sich durch Ausschluss weniger bedroht als wenn er den Auftrag erhielt, mit den Anderen zu spielen. Ähnlich führten die Forscher Walton und Cohen (2011) an einer US-amerikanischen Universität mit Erstsemestern eine kurze Intervention durch, die das Ziel hatte, insbesondere das Zugehörigkeitsgefühl von Minoritätsstudierenden zu stärken. In der Studie lasen die Teilnehmenden Berichte älterer Studierender, die im ersten Jahr häufig Unsicherheit und 
Einsamkeit empfunden hatten, dieser Zustand sich aber über die Zeit verbesserte. Die

Teilnehmenden wurden anschliessend gebeten, einen kurzen Bericht über ähnliche

Erfahrungen zu verfassen, die sie selbst gemacht hatten. Insbesondere bei

Minoritätsstudierenden afroamerikanischer Herkunft zeigten sich noch drei Jahre nach der kurzen Intervention positive Effekte auf Noten, Selbstwert und Gesundheit. Die Forschenden vermuteten, dass der Grund hierfür darin lag, dass die Erstsemester Unsicherheiten und Gefühle der Ablehnung verstärkt auf ihren vorübergehenden Status als Neulinge und nicht auf Diskriminierung aufgrund ihrer ethnischen Herkunft zurückführten. Dies wiederum hatte vermutlich zur Folge, dass sie sich selbstbewusster und zuversichtlicher verhielten und damit mehr Erfolg hatten.

Stärkung des Zugehörigkeitsgefühls. Gegeben die Häufigkeit von Ausgrenzungserlebnissen im Alltag (Nezlek, Wesselmann, Wheeler, \& Williams, 2012), kann es generell sinnvoll sein, das Zugehörigkeitsgefühl der Mitglieder zu einer Organisation zu stärken. Dies hat zum einen den Vorteil, dass kleine Ausgrenzungserlebnisse weniger ins Gewicht fallen, und zum anderen, dass die Mitglieder die Beziehung zu der Organisation als sehr wertvoll betrachten und im Zweifelsfall eher bereit sein werden, diese zu verbessern, anstatt sich zurückzuziehen oder zu kündigen (Smart Richman \& Leary, 2009). Wichtige Massnahmen zur Stärkung der Zugehörigkeit sind das Schaffen eines generell positiven sozialen Klimas und die Förderung von Teamwork (Janke, Nitsche, \& Dickhäuser, 2015; Turner, Barling, \& Zacharatos, 2002). Dabei sollten jedoch zu stark wettbewerbsorientierte Anreize innerhalb der Organisation vermieden werden, sprich: die Mitarbeitenden sollten nicht um Stellen, Gehalterhöhungen, gute Bewertungen oder andere Belohnungen konkurrieren. Auch wenn das Gefühl, mit Anderen in Konkurrenz zu stehen, die Einzelleistung kurzfristig positiv beeinflussen kann (Meece, Anderman, \& Anderman, 2006), so fokussieren Individuen unter diesen Bedingungen vor allem darauf, andere Mitglieder der Organisation auszustechen (Janke et al., 2016). Dieser Fokus allein kann sich bereits 
schädlich auswirken. Hinzu kommt, dass Mitarbeitende sich in einem kompetitiven Klima weniger wohl und zugehörig fühlen (Fletcher, Major, \& Davis, 2008). Stattdessen sollten Führungskräfte sich bemühen ein „Wir“ Gefühl aufzubauen, indem statt der internen eher die externe Konkurrenz zu anderen Organisationen betont wird (Brewer, 1999). Auch soziale Aktivitäten wie beispielsweise gemeinsame Ausflüge, Feste, Teamaktivitäten (z.B. Teilnahme an einem Firmenlauf) können wesentlich dazu beitragen, ein Zugehörigkeitsgefühl zu schaffen (Reis, Sheldon, Gable, Roscoe, \& Ryan, 2000).

Weil die Zugehörigkeit zu einer Mehrheit die negativen Folgen sozialen Ausschlusses reduzieren kann (Eck, et al., in press), könnte es sich für Organisationen allgemein empfehlen, der Entstehung von Randgruppen oder gar Einzelgängern entgegen zu wirken und die Charakteristika tatsächlicher Minderheiten weniger in der Vordergrund zu stellen. Auch auf diese Weise kann das Wir-Gefühl gestärkt werden.

Wertschätzung und Einbezug. Soziale Ausgrenzung bedroht nicht nur die Zugehörigkeit einer Person, sondern auch ihren Selbstwert, sowie das Bedürfnis nach Kontrolle und einer bedeutsamen Existenz (Williams, 2009). Auch hier kann eine Organisation vorbeugen und die entsprechenden Bedürfnisse im Vorfeld stärken. So kann eine Organisation sich entscheiden, die Autonomie und demokratische Teilhabe ihrer Mitglieder zu stärken, sprich, den Mitgliedern der Organisation die Möglichkeit zu geben, eigene Entscheidungen zu treffen und sich einzubringen (Baard, Deci, \& Ryan, 2004). Hierbei ist allerdings zu beachten, dass verstärkte Autonomie trotzdem klare Richtlinien benötigt, da es sonst leicht zu Verwirrung und verstärktem Stress bei den Organisationsmitgliedern kommen kann (Jang, Reeve, \& Deci, 2010). Autonomiefördernde Massnahmen sollten somit stets in einen strukturellen Rahmen eingebunden sein, der eine klare Kommunikation von Erwartungen, Unterstützung und kompetenzförderndes Feedback bietet. In anderen Worten, die Organisationsmitglieder sollten stets das Gefühl haben, dass die Organisation ihren 
Beitrag und ihr Engagement schätzt und unterstützt, und nicht etwa, dass sie mit ihren Entscheidungen allein gelassen werden.

Transparente Informationspolitik. Insbesondere um unbewussten Ausschluss zu vermeiden, sollte eine Organisation stets eine klare, transparente Informationspolitik betreiben und Wert darauf legen, ihre Mitglieder über aktuelle Entwicklungen und Entscheidungen in Kenntnis zu setzen. Dies hat gleich mehrere Vorteile: Fühlen sich die Organisationsmitglieder beachtet und ernstgenommen, erhöht sich oftmals ihre Arbeitszufriedenheit und damit ihre Produktivität (Cotton, et al., 1988). Die Organisation wird zudem von ihren Mitgliedern als positiv wahrgenommen, was die Loyalität steigert und auch die Art und Weise beeinflusst, wie sich Mitglieder gegenüber Anderen über die Organisation äussern (Niehoff, Moorman, Blakely, \& Fuller, 2001). Eine klare Informationspolitik ist aber auch von Vorteil gegenüber Nicht-Organisationsmitgliedern. Häufig kommt es beispielsweise in Bewerbungsverfahren vor, dass nicht ausgewählte Personen nicht einmal eine Absage erhalten. Auch wenn die abgelehnten Bewerbenden sich darüber sicherlich nicht freuen, so schafft eine schriftliche Absage doch zumindest Klarheit und signalisiert zudem, dass die Organisation die Bewerbenden und ihr Anliegen wahrgenommen hat (Rudert, Hales, et al., 2017). Auch im Bereich Service ist es sinnvoll, Kunden, die ein Anliegen oder eine Beschwerde haben, nicht zu ignorieren oder mit elektronischen Protokollen abzufertigen (Williams, 2002): Menschen die sich von einer Organisation wahr- und ernstgenommen fühlen, sind zufriedener und werden im Zweifelsfall auch eher einen negativen Bescheid akzeptieren als Menschen, die das Gefühl haben, dass die Organisation sie ignoriert und ihnen keinen Wert beimisst.

\section{Zusammenfassung}

Ausgrenzung im organisationalen Kontext ist ein häufig auftretendes Phänomen. Oft genügen schon Kleinigkeiten, damit sich Organisationsmitglieder in ihrer Zugehörigkeit bedroht fühlen. Entscheidend ist dabei nicht, ob eine Person objektiv nicht dabei ist, sondern 
wie sie die Situation subjektiv wahrnimmt. Auch Missverständnisse können damit zu Gefühlen der Ausgrenzung führen; und gleichermassen kann eine tatsächliche Ausgrenzung nicht als solche erlebt werden, wenn sie subjektiv anders wahrgenommen wird.

Die Folgen von Ausgrenzung können je nach Intensität und Dauer gravierend sein und reichen von innerlicher bis tatsächlicher Kündigung, über destruktives Arbeitsverhalten bis hin zu gesundheitlichen Risiken wie Depression. Um negative Folgen zu reduzieren, empfehlen sich eine Reihe vorbeugender Massnahmen. Grosse Unternehmen mit hoher Arbeitsbelastung und einer diversen Belegschaft könnten beispielsweise darauf achten, dass sie keinen unbewussten Ausschluss betreiben und nach Möglichkeit Wert auf eine transparente Informationspolitik, demokratischen Einbezug ihrer Mitglieder und Massnahmen legen, welche das Zugehörigkeitsgefühl stärken. Organisationen welche eine starke Fluktuation aufweisen, könnten beispielsweise Wert darauf legen, dass die organisationsinternen Regeln und Normen klar an Neuankömmlinge kommuniziert werden, damit diese normkonformen Ausschluss nicht fehlinterpretieren. Zuletzt empfiehlt es sich generell, Organisationsmitglieder in Trainings und Vorträgen über die negativen Folgen von sozialem Ausschluss zu informieren und ein inklusives Organisationsklima zu schaffen, welches unterschiedliche Ansichten unterstützt und die Mitglieder darin bestätigt, auch kritische Meinungen zu äussern. 


\section{Literaturverzeichnis}

Asch, S. E. (1956). Studies of independence and conformity: I. A minority of one against a unanimous majority. Psychological Monographs: General and Applied, 70(9), 1-70.

Baard, P. P., Deci, E. L., \& Ryan, R. M. (2004). Intrinsic need satisfaction: A motivational basis of performance and weil-being in two work settings. Journal of Applied Social Psychology, 34(10), 2045-2068.

Baumeister, R. F., \& Leary, M. R. (1995). The need to belong: Desire for interpersonal attachments as a fundamental human motivation. Psychological Bulletin, 117(3), 497529. doi: 10.1037/0033-2909.117.3.497

Brewer, M. B. (1999). The psychology of prejudice: Ingroup love and outgroup hate? Journal of Social Issues, 55(3), 429-444.

Cotton, J. L., Vollrath, D. A., Froggatt, K. L., Lengnick-Hall, M. L., \& Jennings, K. R. (1988). Employee participation: Diverse forms and different outcomes. Academy of Management Review, 13(1), 8-22.

Eck, J., Schoel, C., \& Greifeneder, R. (2016). Coping with or buffering against the negative impact of social exclusion on basic needs: A review of strategies. In P. Riva \& J. Eck (Eds.), Social exclusion: Psychological approaches to understanding and reducing its impact (pp. 227-249). New York, NY: Springer.

Eck, J., Schoel, C., \& Greifeneder, R. (in press). Belonging to a Majority Reduces the Immediate Need Threat from Ostracism in Individuals with a High Need to Belong. European Journal of Social Psychology.

Eisenberger, N. I., Lieberman, M. D., \& Williams, K. D. (2003). Does rejection hurt? An fMRI study of social exclusion. Science, 302(5643), 290-292. doi: 10.1126/science. 1089134

Feldman, D. C., Doerpinghaus, H. I., \& Turnley, W. H. (1994). Managing temporary workers: A permanent HRM challenge. Organizational Dynamics, 23(2), 49-63.

Ferris, D. L., Brown, D. J., Berry, J. W., \& Lian, H. (2008). The development and validation of the Workplace Ostracism Scale. Journal of Applied Psychology, 93(6), 1348-1366. doi: $10.1037 / \mathrm{a} 0012743$

Fletcher, T. D., Major, D. A., \& Davis, D. D. (2008). The interactive relationship of competitive climate and trait competitiveness with workplace attitudes, stress, and performance. Journal of Organizational Behavior, 29(7), 899-922. 
Gkorezis, P., \& Bellou, V. (2016). The relationship between workplace ostracism and information exchange: The mediating role of self-serving behavior. Management Decision, 54(3), 700-713.

Gkorezis, P., Panagiotou, M., \& Theodorou, M. (2016). Workplace ostracism and employee silence in nursing: The mediating role of organizational identification. Journal of Advanced Nursing, 72(10), 2381-2388.

Golden, T. D., Veiga, J. F., \& Dino, R. N. (2008). The impact of professional isolation on teleworker job performance and turnover intentions: Does time spent teleworking, interacting face-to-face, or having access to communication-enhancing technology matter? Journal of Applied Psychology, 93(6), 1412-1421.

Hales, A. H., Kassner, M. P., Williams, K. D., \& Graziano, W. G. (2016). Disagreeableness as a cause and consequence of ostracism. Personality and Social Psychology Bulletin, 42(6), 782-797. doi: 10.1177/0146167216643933

Hales, A. H., Williams, K. D., \& Rector, J. (2017). Alienating the audience: How abbreviations hamper scientific communication. Observer, 30, 22-24.

Hitlan, R. T., Kelly, K. M., Schepman, S., Schneider, K. T., \& Zárate, M. A. (2006). Language exclusion and the consequences of perceived ostracism in the workplace. Group Dynamics: Theory, Research, and Practice, 10(1), 56-70.

Hitlan, R. T., \& Noel, J. (2009). The influence of workplace exclusion and personality on counterproductive work behaviours: An interactionist perspective. European Journal of Work and Organizational Psychology, 18(4), 477-502.

Jang, H., Reeve, J., \& Deci, E. L. (2010). Engaging students in learning activities: It is not autonomy support or structure but autonomy support and structure. Journal of Educational Psychology, 102(3), 588-600.

Janke, S., Nitsche, S., \& Dickhäuser, O. (2015). The role of perceived need satisfaction at work for teachers' work-related learning goal orientation. Teaching and Teacher Education, 47, 184-194.

Janke, S., Nitsche, S., Praetorius, A.-K., Benning, K., Fasching, M., Dresel, M., \& Dickhäuser, O. (2016). Deconstructing performance goal orientations: The merit of a dimensional approach. Learning and Individual Differences, 50, 133-146.

Jones, E. E., Carter-Sowell, A. R., Kelly, J. R., \& Williams, K. D. (2009). 'I'm out of the loop': Ostracism through information exclusion. Group Processes \& Intergroup Relations, 12(2), 157-174. doi: 10.1177/1368430208101054 
Korte, R. F. (2009). How newcomers learn the social norms of an organization: A case study of the socialization of newly hired engineers. Human Resource Development Quarterly, 20(3), 285-306.

Lustenberger, D. E., \& Jagacinski, C. M. (2010). Exploring the effects of ostracism on performance and intrinsic motivation. Human Performance, 23(4), 283-304.

Meece, J. L., Anderman, E. M., \& Anderman, L. H. (2006). Classroom goal structure, student motivation, and academic achievement. Annual Review of Psychology, 57, 487-503.

Mesmer-Magnus, J. R., \& Viswesvaran, C. (2005). Whistleblowing in organizations: An examination of correlates of whistleblowing intentions, actions, and retaliation. Journal of Business Ethics, 62(3), 277-297.

Miceli, M. P., \& Near, J. P. (1989). The incidence of wrongdoing, whistle-blowing, and retaliation: Results of a naturally occurring field experiment. Employee Responsibilities and Rights Journal, 2(2), 91-108.

Miceli, M. P., Near, J. P., \& Dworkin, T. M. (2009). A word to the wise: How managers and policy-makers can encourage employees to report wrongdoing. Journal of Business Ethics, 86(3), 379-396.

Nezlek, J. B., Wesselmann, E. D., Wheeler, L., \& Williams, K. D. (2012). Ostracism in everyday life. Group Dynamics: Theory, Research, and Practice, 16(2), 91-104. doi: $10.1037 / \mathrm{a} 0028029$

Niehoff, B. P., Moorman, R. H., Blakely, G., \& Fuller, J. (2001). The influence of empowerment and job enrichment on employee loyalty in a downsizing environment. Group \& Organization Management, 26(1), 93-113.

O'Reilly, J., Robinson, S. L., Berdahl, J. L., \& Banki, S. (2015). Is negative attention better than no attention? The comparative effects of ostracism and harassment at work. Organization Science, 26(3), 774-793. doi: 10.1287/orsc.2014.0900

Parmerlee, M. A., Near, J. P., \& Jensen, T. C. (1982). Correlates of whistle-blowers' perceptions of organizational retaliation. Administrative Science Quarterly, 27(1), 1734. doi: $10.2307 / 2392544$

Pinto, I. R., Marques, J. M., Levine, J. M., \& Abrams, D. (2010). Membership status and subjective group dynamics: Who triggers the black sheep effect? Journal of Personality and Social Psychology, 99(1), 107-119.

Reis, H. T., Sheldon, K. M., Gable, S. L., Roscoe, J., \& Ryan, R. M. (2000). Daily well-being: The role of autonomy, competence, and relatedness. Personality and Social Psychology Bulletin, 26(4), 419-435. doi: 10.1177/0146167200266002 
Riva, P., Montali, L., Wirth, J. H., Curioni, S., \& Williams, K. D. (2016). Chronic social exclusion and evidence for the resignation stage: An empirical investigation. Journal of Social and Personal Relationships, 1-24.

Robinson, S. L., O’Reilly, J., \& Wang, W. (2013). Invisible at work: An integrated model of workplace ostracism. Journal of Management, 39(1), 203-231.

Rudert, S. C., \& Greifeneder, R. (2016). When it's okay that I don't play: Social norms and the situated construal of social exclusion. Personality and Social Psychology Bulletin, 42(7), 955-969. doi: 10.1177/0146167216649606

Rudert, S. C., Hales, A. H., Greifeneder, R., \& Williams, K. D. (2017). When silence is not golden: Why acknowledgement matters even when being excluded. Personality and Social Psychology Bulletin. doi: 10.1177/0146167217695554

Rudert, S. C., Janke, S., \& Greifeneder, R. (2017). Under threat by popular vote: Germanspeaking immigrants' affect and cognitions following the Swiss vote against mass immigration. PLOS ONE, 12(4). doi: 10.1371/journal.pone.0175896.

Rudert, S. C., Reutner, L., Greifeneder, R., \& Walker, M. (2017). Faced with exclusion: Perceived facial warmth and competence influence moral judgments of social exclusion. Journal of Experimental Social Psychology, 68, 101-112. doi: 10.1016/j.jesp.2016.06.005

Saks, A. M. (1994). Moderating effects of self-efficacy for the relationship between training method and anxiety and stress reactions of newcomers. Journal of Organizational Behavior, 15(7), 639-654.

Schoel, C., Eck, J., \& Greifeneder, R. (2014). A matter of vertical position: Consequences of ostracism differ for those above versus below its perpetrators. Social Psychological and Personality Science, 5(2), 149-157 doi: 10.1177/1948550613488953

Scott, K. L., Restubog, S. L. D., \& Zagenczyk, T. J. (2013). A social exchange-based model of the antecedents of workplace exclusion. Journal of Applied Psychology, 98(1), 3748.

Smart Richman, L., \& Leary, M. R. (2009). Reactions to discrimination, stigmatization, ostracism, and other forms of interpersonal rejection: A multimotive model. Psychological Review, 116(2), 365-383. doi: 10.1037/a0015250

Spector, P. E., \& Jex, S. M. (1998). Development of four self-report measures of job stressors and strain: Interpersonal Conflict at Work Scale, Organizational Constraints Scale, Quantitative Workload Inventory, and Physical Symptoms Inventory. Journal of Occupational Health Psychology, 3(4), 356 - 367. 
Turner, N., Barling, J., \& Zacharatos, A. (2002). Positive psychology at work. In C. R. Snyder \& S. J. Lopez (Eds.), Handbook of Positive Psychology (pp. 715 - 728). New York, NY: Oxford University Press.

von Hippel, C., Mangum, S. L., Greenberger, D. B., Heneman, R. L., \& Jeffrey, D. S. (1997). Temporary employment: Can organizations and employees both win? The Academy of Management Executive 11(1), 93-104.

Walton, G. M., \& Cohen, G. L. (2011). A brief social-belonging intervention improves academic and health outcomes of minority students. Science, 331(6023), 1447-1451. doi: $10.1126 /$ science. 1198364

Wesselmann, E. D., Cardoso, F. D., Slater, S., \& Williams, K. D. (2012). To be looked at as though air: Civil attention matters. Psychological Science, 23(2), 166-168. doi: $10.1177 / 0956797611427921$

Wesselmann, E. D., Williams, K. D., \& Hales, A. H. (2013). Vicarious ostracism. Frontiers in Human Neuroscience, 7. doi: 10.3389/fnhum.2013.00153

Wesselmann, E. D., Wirth, J. H., Pryor, J. B., Reeder, G. D., \& Williams, K. D. (2013). When do we ostracize? Social Psychological and Personality Science, 4(1), 108-115. doi: $10.1177 / 1948550612443386$

Williams, K. D. (2002). Ostracism: The power of silence. New York, NY: Guilford Press.

Williams, K. D. (2009). Ostracism: A temporal need-threat model. In P. Z. Mark (Ed.), Advances in Experimental Social Psychology (Vol. 41, pp. 275-314). San Diego, CA: Elsevier Academic Press.

Williams, K. D., \& Nida, S. A. (2014). Ostracism and Public Policy. Policy Insights from the Behavioral and Brain Sciences, 1(1), 38-45.

Wu, L., Wei, L., \& Hui, C. (2011). Dispositional antecedents and consequences of workplace ostracism: An empirical examination. Frontiers of Business Research in China, 5(1), $23-44$. 